\title{
Mycoplasma genitalium: the next sexually transmitted superbug?
}

The wrong email address was given for the corresponding author in this editorial by Gwenda Hughes and John Saunders (BMJ 2018;363:k4376, doi:10.1136/bmj.k4376). It has been amended online. 